\title{
Treatment of Ewing sarcoma in children: Results from a single centre
}

\section{Dear Editor,}

Ewing sarcoma is a malignant mesenchymal tumour that presents as a bone or soft-tissue sarcoma. Translocations involving the EWS gene on chromosome 22q12 are unique molecular signatures. ${ }^{1,2}$ Compared with the West where the annual incidence is $1-3$ per million, incidence has been reported to be lower among Asians ${ }^{1}$ and in East Asia. ${ }^{3}$ Paediatric-specific management and outcome data are distinctively absent in this region, which prompted the report of our experience herein.

This is a retrospective chart review of all paediatric patients treated for Ewing sarcoma from 2008 to 2020 at our institution, the Mount Elizabeth Hospital in Singapore. Their clinical, histopathological, treatment and follow-up data were summarised. Survival was measured from diagnosis and censored at the end of May 2021 or upon death. A literature search from PubMed was conducted using the search terms: ((Ewing sarcoma) AND (child OR pediatric)) AND (China OR Hong Kong OR Japan OR Korea OR Taiwan OR Thailand OR Singapore) AND Treatment. These countries/territories were selected as they represented the regions where childhood oncology studies were most often reported in East Asia and Southeast Asia. The search results were screened to include only clinical studies with at least 10 subjects involving children ( $\leq 18$ years old). Approval from institutional review board was not required for observational studies outside the context of a clinical trial in our institution.

Ten children were treated for histopathology confirmed Ewing sarcoma during the study period (Table 1), with mean age of 7.0 (range 2.2-16.8) years. Eight (80\%) of the children were boys. EWS-FLII transcript was tested by fluorescence in situ hybridisation in 8 cases and all were positive. Eight had skeletal primaries (femur, 4; chest wall, 2; tibia, 1; skull base, 1), while 2 had lung tumour as the extraskeletal primary. Seven children presented with localised disease while 3 had metastatic disease. All patients were from Asia (Bahrain, 2; Bangladesh, 1; China 4; Singapore, 1; Vietnam, 2). Seven patients were treatment naive while 3 had received neoadjuvant chemotherapy from other centres before they came to our hospital for surgery and postoperative management. At a median follow-up of 3.1 (range $0.5-12.9$ ) years, 9 were surviving in first complete remission and 1 died of the disease, yielding an overall and event-free survival of $88.9 \%$.
Six children (localised, 4; metastatic, 2) were treated according to the European Ewing tumour Working Initiative of National Groups (Euro-E.W.I.N.G.) protocol with 5 cycles of vincristine, ifosfamide, doxorubicin and etoposide (VIDE) as induction chemotherapy. Complete resection of primary tumour was achieved in 1 patient at diagnosis, 4 after neoadjuvant therapy, while the 1 patient with skull base tumour had complete resolution of the disease on chemotherapy alone. Among the 4 surgical specimens obtained after chemotherapy, 2 had tumour necrosis of $90-99 \%$ and the other 2 had complete necrosis. All 6 patients were surviving in complete remission.

Four children received neoadjuvant chemotherapy according to the US Children's Oncology Group (COG) protocol with vincristine, doxorubicin, cyclophosphamide, and alternating with ifosfamide and etoposide (VDCIE) on 2-week cycles. Histopathology showed tumour necrosis of $<90 \%$ in all cases. Three of them were surviving, with 2 in complete remission and 1 with disease in metabolic remission. The other patient relapsed and died at 1.1 years after diagnosis.

The literature search done in May 2021 obtained 280 articles. Eleven studies were finally identified (materials available on request). All were retrospective studies. They originated from China $(n=3)$, Japan $(n=5)$, Korea $(n=1)$ and Taiwan $(n=2)$. None was reported from Hong Kong, Singapore or Thailand. None of the studies was specific for children, and all were combined paediatric and adult series. All followed international protocols with combined modality treatment comprising all 3 modalities: chemotherapy, surgery and radiotherapy. The chemotherapy regimens used were quite variable even within the same studies, but VDC-IE appeared to be the most popular regimen. Survival rates were $5.9-78.3 \%$. Notably, from the 2 largest series of publications, younger patients fared better in terms of long-term event-free survival.,7

Prior to the era of chemotherapy, $90 \%$ of patients with localised Ewing sarcoma died from the disease after local therapy alone. ${ }^{1}$ Hence, systemic chemotherapy and local treatment (surgery and/or radiotherapy) are both indispensable in the contemporary management of Ewing sarcoma. Prognostic factors are influenced by age, tumour extent or volume, stage of the disease, success of local therapy, but histological response with $\geq 90 \%$ of tumour necrosis is the strongest predictor for long- 


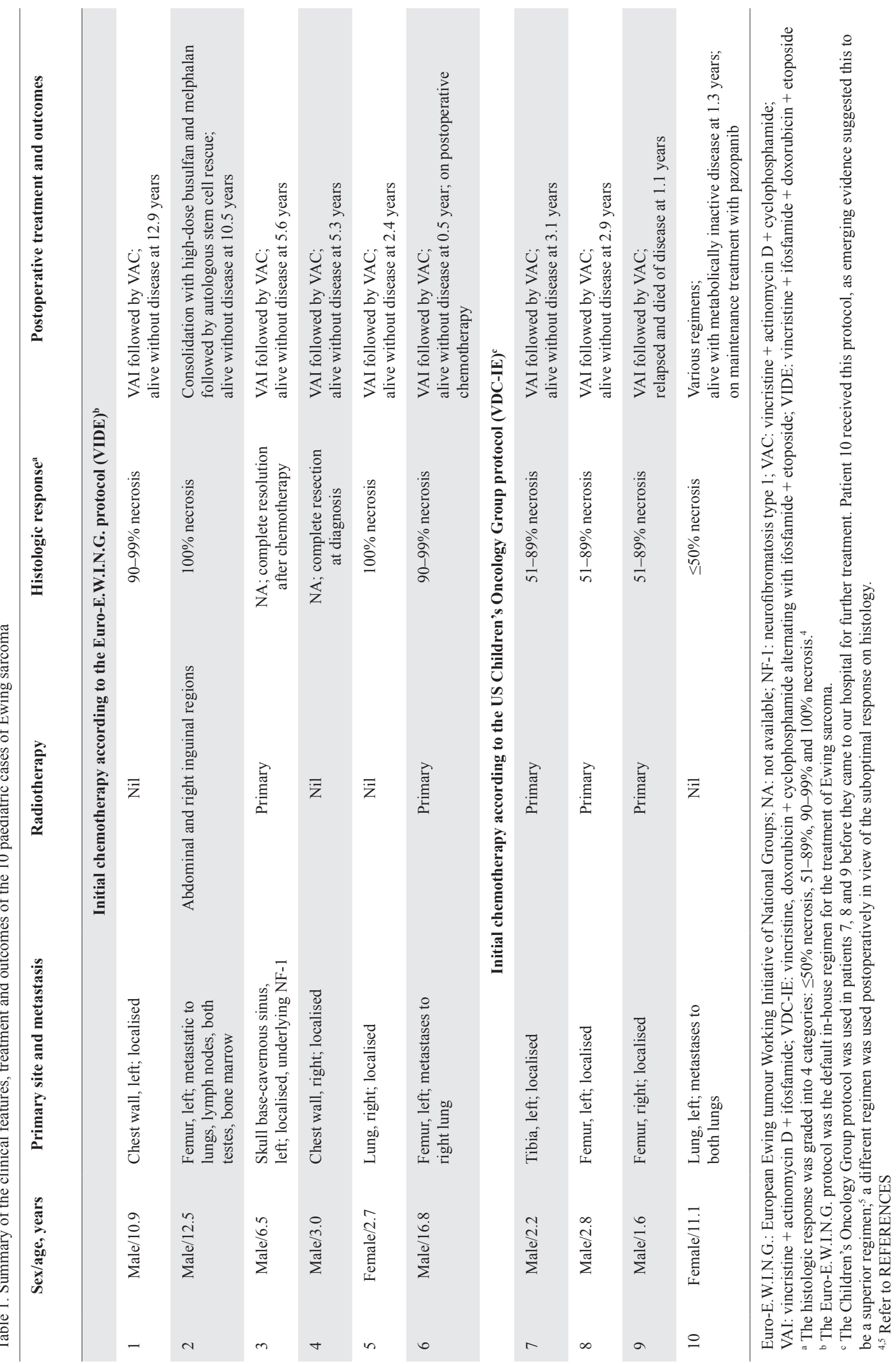


term survival. ${ }^{2}$ Hence, the importance of an effective chemotherapeutic regimen cannot be overemphasised.

Current chemotherapeutic regimens that are highly effective for Ewing sarcoma are pioneered by intergroup collaborations in both Europe and North America. The Euro-E.W.I.N.G. 99 was the first intergroup regimen in Europe. The protocol uses vincristine, ifosfamide, doxorubicin, etoposide, cyclophosphamide and dactinomycin in various combinations at 3-week intervals over 42 weeks as the treatment backbone. ${ }^{8}$ The COG protocol uses vincristine, doxorubicin and cyclophosphamide, alternating with ifosfamide and etoposide (VDC-IE) at 2-week intervals over 28 weeks. ${ }^{9}$ However, the $\mathrm{COG}$ chemotherapeutic regimen has been considered too harsh in some Asian countries. ${ }^{10}$ Among the studies identified from the literature search that mentioned the use of VDC-IE, ${ }^{11,12}$ the alternating cycles were administered at 3 -week intervals, with a total duration of treatment of 42 weeks. This might have been one of the contributing factors for the generally inferior treatment outcomes observed in Asian patients with Ewing sarcoma, which deserves further study.

From our experience, based on the extent of tumour necrosis after induction treatment, the Euro-E.W.I.N.G. regimen seems to be equally effective in Asia compared with the COG protocol. However, the number of patients is small. More studies from East Asia and Southeast Asia are needed to inform paediatric oncologists on the optimal management of childhood Ewing sarcoma, including the level of supportive care required. Given the rarity of the disease in this part of the world, international collaboration is clearly indicated.

\section{REFERENCES}

1. Hesla AC, Papakonstantinou A, Tsagkozis P. Current status of management and outcome for patients with Ewing sarcoma. Cancers (Basel) 2021;13:1202.

2. DuBois SG, Dirksen U. Contemporary approach to therapy for Ewing sarcoma. In: Arndt CA (Ed). Sarcomas of bone and soft tissues in children and adolescents. 1st Ed. Cham: Springer Nature Switzerland AG; 2021.

3. Fukushima T, Ogura K, Akiyama T, et al. Descriptive epidemiology and outcomes of bone sarcomas in adolescent and young adult patients in Japan. BMC Musculoskelet Disord 2018;19:297.
4. Wunder JS, Paulian G, Huvos AG, et al. The histological response to chemotherapy as a predictor of the oncological outcome of operative treatment of Ewing sarcoma. J Bone Joint Surg Am 1998;80:1020-33.

5. Wheatley K, Moroz V, Marec-Berard, et al. First results of the Euro Ewing 2012 trial comparing two chemotherapy regimens in newly diagnosed Ewing sarcoma. Presented at the Connective Tissue Oncology Society Annual Meeting, 13-16 November 2019, Tokyo, Japan.

6. Obata H, Ueda T, Kawai A, et al. Clinical outcome of patients with Ewing sarcoma family of tumors of bone in Japan: the Japanese Musculoskeletal Oncology Group Cooperative Study. Cancer 2007; 109:767-75.

7. Lee JA, Kim DH, Cho J, et al. Treatment outcome of Korean patients with localized Ewing sarcoma family of tumors: a single institution experience. Jpn J Clin Oncol 2011;41:776-782.

8. Le Deley MC, Paulussen M, Lewis I, et al. Cyclophosphamide compared with ifosfamide in consolidation treatment of standardrisk Ewing sarcoma: results of the randomized noninferiority Euro-EWING99-R1 trial. J Clin Oncol 2014;32:2440-8.

9. Womer RB, West DC, Krailo MD, et al. Randomized controlled trial of interval-compressed chemotherapy for the treatment of localized Ewing sarcoma: a report from the Children's Oncology Group. J Clin Oncol 2012;30:4148-54.

10. Totadri S, Bansal D, Rao KLN, et al. Challenges in the management of localized Ewing sarcoma in a developing country. Pediatr Hematol Oncol 2020;37:610-9.

11. Xie CF, Liu MZ, Xi M. Extraskeletal Ewing's sarcoma: a report of 18 cases and literature review. Chin J Cancer 2010;29:420-4.

12. Lee CY, Yen CC, Yen HJ, et al. Outcomes of 50 patients with Ewing sarcoma family of tumors treated at a single institution in Taiwan. Medicine 2016;95:e3830.

Anselm Chi-Wai Lee ${ }^{\llbracket 1}{ }_{\text {FAMS }}$, Saminathan Suresh Nathan ${ }^{2}$ FAMS, Chan Hon Chui ${ }^{\beta} F R C S$, Kim Shang $\underline{\text { Leel }}^{4}$ FAMS

${ }^{1}$ Children's Haematology and Cancer Centre, Mount Elizabeth Hospital, Singapore

${ }^{2}$ Limb Salvage and Revision Arthroplasty Surgery, Mount Elizabeth Medical Centre, Singapore

${ }^{3}$ Surgery Centre for Children, Mount Elizabeth Medical Centre, Singapore

${ }^{4}$ Radiation Oncology Centre, Mount Elizabeth Hospital, Singapore

Correspondence: Dr Anselm Chi-Wai Lee, Children's Haematology and Cancer Centre, Mount Elizabeth Hospital, Level 4, 3 Mount Elizabeth, Singapore 228510 .

Email: anselm.cw.lee@gmail.com 